\title{
La industria de la música en España
}

\author{
Juan C. Calvi ${ }^{1}$ \\ Universidad Rey Juan Carlos de Madrid
}

Resumen: El presente artículo analiza la estructura de la industria de la música en España y las estrategias que las compañías discográficas están desarrollando actualmente. Analiza además el fenómeno social de reproducción y descarga gratuita de música (tanto en Internet como fuera de ella) denominado comúnmente como "piratería musical", y de qué modo las compañías discográficas están reaccionando frente a este problema.

Palabras claves: comunicación; industria de la música; piratería; Internet.

Abstract: The present article analyses the Spanish music industry and the strategies currently been developed by the recording industry. It also analyses the social phenomenon of free music reproduction and download (both in the Internet, and outside) commonly called "musical piracy", and how the recording companies are facing this problem.

Key words: communication; music industry; piracy; Internet.

Resumo: $O$ presente artigo analisa a estrutura da indústria da música na Espanha e as estratégias que as gravadoras estão desenvolvendo atualmente. Examina, também, o fenômeno social de reprodução e download gratuito de música (tanto na Internet quanto fora dela) denominado comumente de "pirataria musical", e, de que modo, as gravadoras estão reagindo a este problema.

Palavras-chave: comunicação; indústria da música; pirataria; Internet.

\footnotetext{
1 Es Profesor Titular (I) de la Universidad Rey Juan Carlos de Madrid. Doctor en Ciencias de la Información por la Universidad Complutense de Madrid, ha realizado postgrados y estancias de investigación en las universidades de Boloña, Rosario, Río do Janeiro, Sao Paulo y Sergipe, y es profesor visitante de la Universidad de Westminster. Entre sus publicaciones se destacan: La circulación de productos audiovisuales en Internet. Análisis económico, político y social de la reproducción cultural en la Era Digital (Madrid: Ed. Dykinson, 2006, en prensa); ¿Reproducción de la cultura o cultura de la reproducción? Hacia un nuevo régimen de regulación y explotación de productos culturales en Internet, en F. Sierra, C. Bolaño y G. Mastrini (Eds.), Economía Política, Comunicación y Conocimiento: una perspectiva crítica latinoamericana (Buenos Aires: La Crujía, 2005).
} 
La industria de la música constituye hoy uno de los sectores más potentes y de mayor crecimiento de la economía mundial, no sólo como un sector particular dentro del conjunto de sectores que componen las llamadas Industrias Culturales (I.C.), sino también como irrigador de productos que son utilizados por el resto de estos sectores, tales como la radio y la televisión, la industria cinematográfica y de videojuegos, etc., y también como componente fundamental de otros sectores industriales tales como la electrónica de consumo, la publicidad, las telecomunicaciones, etc.

Concretamente en España, en 2003 la industria de la música generó directamente un volumen de negocio de $1.191,8$ millones de euros y dio empleo a 52.850 personas; y tomando en cuenta el negocio de la música como componente de otros sectores de la economía tales como la electrónica de consumo, los medios de comunicación y la publicidad, las telecomunicaciones, bares, discotecas y salas de baile, etc., el volumen de negocio total que generó la música en España en el mismo año fue de 4.564,9 millones de euros o el equivalente al 0.8\% del PIB (IFPI, 2005b; Promusicae, 2005).

Específicamente en el sector de los medios de comunicación, las denominadas "radio fórmulas" o programas de radio dedicados exclusivamente a la música constituyen el principal medio de promoción de la industria musical, conjuntamente con los programas de televisión con contenido musical. En España, en 2003, el número de oyentes de las radio fórmulas fue de 9,8 millones de personas, según datos del Estudio General de Medios (EGM) para ese año. A partir de estos datos, se estima que la cifra de negocio de las radio fórmulas españolas en ese año ascendió a 149,7 millones de euros. En relación al sector de la televisión, los contenidos musicales en la televisión ocupan aproximadamente un 3.6\% del total de las emisiones, por lo que se calcula que el impacto inducido de la música en la televisión es de 163,8 millones de euros (Promusicae, 2005).

En 2004, tomando en cuenta sólo el mercado de los CD musicales, se vendieron 50 millones de unidades y se facturaron 482,47 millones de euros. Ahora bien, según el último informe elaborado por la SGAE (Sociedad General de Autores y Editores de España) sobre la situación del mercado de la música en España (SGAE, 2005), en los 
últimos años la venta de fonogramas en todos los soportes ha caído un $36.99 \% \%^{2}$, pasando de 77,8 millones de unidades en el año 2000 a 49,1 millones en el 2004, donde el soporte CD es el que más ha acusado esta caída, pasando de 67,3 millones de unidades a 44,6 millones en el mismo período.

Las causas de esta caída apuntan principalmente a la piratería de los CD y la copia privada de los mismos, más las descargas de música en Internet. No obstante, para analizar esta situación se deben tomar en cuenta otros factores determinantes, principalmente el reemplazo del soporte CD por los nuevos soportes digitales y el despegue de los servicios de comercialización de música a través de Internet.

Aún así, el mercado de la música sigue en plena actividad, y en este sentido es necesario señalar que la SGAE ha conseguido durante 2004 la mayor recaudación de su historia al alcanzar 300,7 millones de euros de ingresos, lo que se ha traducido en un aumento del $11.9 \%$ respecto al ejercicio anterior; y de los cuales un $20.9 \%$ se ha recaudado por la polémica imposición del pago de un canon por los CD y DVD vírgenes. Asimismo las artes escénicas y musicales generaron un 10.8\% más de recaudación que en el ejercicio anterior, los espectáculos en vivo un 8.8\% y el apartado de radio y TV un 6.1\% (SGAE, 2004).

El mercado discográfico español ocupa la novena posición en el mercado internacional en términos de volumen de facturación, lo que equivale al 1.9\% del mercado discográfico mundial (IFPI, 2005b). Sin embargo, la industria de la música tanto a nivel mundial como a nivel nacional está controlada por cuatro grandes compañías discográficas multinacionales (Majors), y la principal consecuencia de esta situación es una tendencia general hacia la homogeneización de los mercados musicales tanto internacionales como nacionales.

La estrategia de estas empresas en los mercados nacionales es promover la venta de su repertorio internacional, así como también producir y distribuir artistas locales, compitiendo en este mercado con las Pequeñas y Medianas Empresas Discográficas nacionales (PYMED, también llamadas Indies). Asimismo, las Majors tienen poder de acceso

\footnotetext{
${ }^{2}$ Estas unidades comprenden los soportes físicos LP, Single, cassete, CD y DVD musical. 
a los medios de comunicación, principalmente la radio, utilizando una gran cantidad de recursos publicitarios y de marketing para promocionar sus productos musicales, desplazando así a las empresas más pequeñas y creando barreras de entrada a los nuevos sellos discográficos locales, alternativos o minoritarios, tal como veremos en relación al mercado discográfico español.

\section{La estructura del mercado discográfico español}

El proceso de concentración del mercado de la música en España fue mucho más rápido que el del mercado internacional. En 1980 siete compañías discográficas internacionales controlaban el 52\% del mercado nacional, mientras que en 1985 eran solo cinco discográficas las que concentraban el 87\% del mercado (Buquet, 2002); y cuando analizamos la estructura actual del mercado discográfico en España, vemos que éste es un reflejo a pequeña escala del mercado discográfico internacional, tal como muestra el siguiente gráfico:

Estructura del mercado discográfico en España, 2005

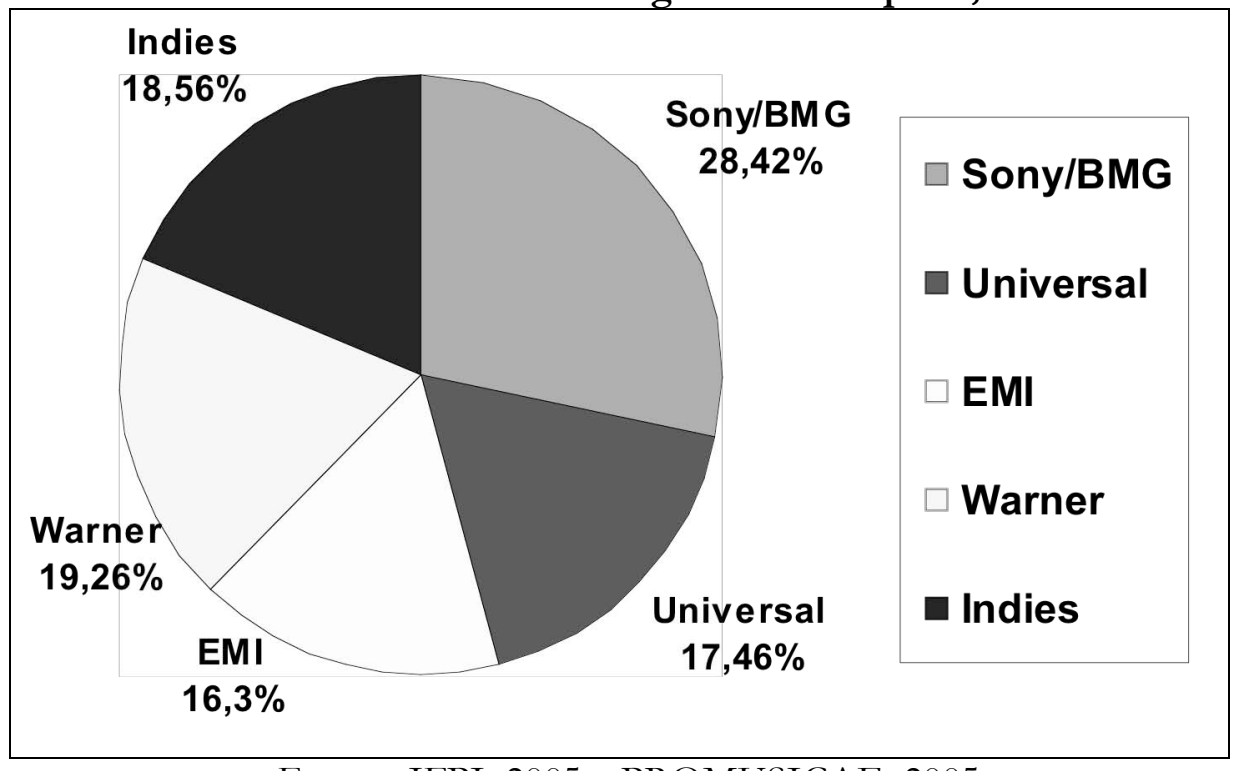

Fuente: IFPI, 2005 y PROMUSICAE, 2005 
Actualmente, las compañías discográficas multinacionales que acaparan más del $80 \%$ del mercado discográfico español son: Sony BMG con el $28.42 \%$, Universal con el $17.46 \%$, seguida de Warner con el $19.26 \%$ y EMI con el $16.3 \%$. El 18.56\% restante se reparte entre las Indies nacionales (IFPI, 2005 y PROMUSICAE, 2005).

Como hemos comentado, la estrategia comercial de las Majors consiste en la explotación masiva de los repertorios musicales internacionales y también nacionales, estableciendo filiales locales en aquellos mercados discográficos más importantes y lucrativos, produciendo artistas locales, compitiendo con los sellos nacionales y desarrollando lanzamientos internacionales.

Ahora bien, las Majors y las PYMED han desarrollado una relación funcional en la que estas últimas operan como viveros creativos de las primeras, aunque ello no significa necesariamente que el repertorio nacional esté relegado a un porcentaje marginal del mercado, sino más bien que la explotación de éste queda en manos de las principales compañías discográficas.

Esto puede comprobarse comparando la distribución entre repertorio nacional y repertorio internacional en el mercado discográfico español con la distribución de los mismos repertorios en los diez principales mercados discográficos del mundo; y así tenemos que en España el porcentaje del repertorio nacional se encuentra en la media de estos países, en torno al 50\% del mercado, tal como muestra la siguiente tabla:

Porcentaje de los repertorios nacionales en los 10 principales mercados discográficos del mundo, 2003

\begin{tabular}{|c|c|c|}
\hline Posición & País & $\begin{array}{c}\text { Porcentaje del repertorio } \\
\text { nacional }\end{array}$ \\
\hline 1 & EE.UU. & $93 \%$ \\
\hline 2 & Japón & $72 \%$ \\
\hline 3 & Francia & $60 \%$ \\
\hline 4 & Alemania & $48 \%$ \\
\hline 5 & Italia & $48 \%$ \\
\hline 6 & Reino Unido & $47 \%$ \\
\hline 7 & España & $46 \%$ \\
\hline 8 & Australia & $26 \%$ \\
\hline 9 & Canadá & $22 \%$ \\
\hline 10 & Holanda & $19 \%$ \\
\hline
\end{tabular}

Fuente: OCDE, 2005 
Del análisis de estos datos se desprende que las Majors no solo comercializan sus repertorios internacionales sino que desarrollan una estrategia de explotación de los repertorios musicales nacionales a través de sus filiales locales, compitiendo pero a la vez alimentándose de las PYMED en su fase de exploración, descubrimiento y producción de los artistas e intérpretes locales. Y a pesar de esta relación funcional entre Majors internacionales y PYMED nacionales, en el transcurso de los últimos años los sellos discográficos nacionales han ido desapareciendo, ocasionando un deterioro en el tejido de la industria de la música en España y una tendencia hacia la homogeneización del mercado de la música local.

\section{La creciente desaparición de los sellos discográficos nacionales}

La caída de las ventas de música en el mercado internacional y su repercusión en el mercado español han acelerado el proceso de concentración de las empresas discográficas que ya se venía anunciando desde la década de los años 80. Sumado a la estrategia de las Majors para establecer filiales locales y explotar los repertorios nacionales, el resultado ha sido que los sellos discográficos nacionales se han visto vaciados de contenidos y han sido absorbidos por las grandes compañías o han tendido a desaparecer. No obstante, conjuntamente a este proceso, se ha producido la aparición de pequeños sellos independientes, minoritarios o alternativos, más especializados en la búsqueda de nuevos talentos y más arriesgados en sus apuestas discográficas.

Durante los años 80, las compañías discográficas de capital nacional más importantes fueron absorbidas por las Majors o fueron desapareciendo, mientras iban apareciendo nuevos sellos independientes; y durante los años 90 y a comienzos del 2000 esta tendencia se reforzó.

Si observamos la evolución de la cantidad de sellos discográficos de acuerdo a la cantidad de discos que se han editado por año, entre los años 2000 y 2004, vemos que los sellos que editan menos de 30 discos al año han pasado de 554 a 669 en ese período, los que editan entre 30 y 200 discos al año han pasado de 71 a 60, y los sellos que editan más 
de 200 discos al año han pasado de 15 a 13, tal como muestra la siguiente tabla (SGAE, 2005):

Evolución de las compañías discográficas en España según el número de discos editados, 2000-2004

\begin{tabular}{|l|c|c|c|c|c|}
\hline $\begin{array}{c}\text { Discos } \\
\text { editados/Año }\end{array}$ & 2000 & 2001 & 2002 & 2003 & 2004 \\
\hline Más de 200 & 15 & 17 & 19 & 17 & 13 \\
\hline De 30 a 200 & 71 & 60 & 67 & 60 & 60 \\
\hline Menos de 30 & 554 & 574 & 595 & 639 & 669 \\
\hline
\end{tabular}

Fuente: SGAE, 2005

Esta evolución demuestra que el vivero de sellos independientes ha crecido, los sellos de mediana escala han disminuido, y los grandes sellos discográficos se han concentrado; es decir, fueron desapareciendo las empresas discográficas nacionales que podían competir con las Majors y aumentaron los pequeños sellos independientes que no pueden competir con ellas pero sí alimentarlas en la fase de producción.

Actualmente, como vimos en el primer gráfico, las Majors controlan el $81.44 \%$ del mercado nacional, mientras que las PYMED de capital español se quedan con el 18.56\% del mercado restante. Esto se ve reflejado en la lista de los discos más vendidos en España, y si tomamos como referencia la lista de los discos más vendidos (febrero de 2006), tenemos que las empresas que controlan la mayor parte de los lanzamientos discográficos en el mercado nacional son Sony BMG (cuatro discos), EMI (dos discos) y Warner (un disco).

En relación a la evolución de los precios de los CD en España, al igual que en la mayoría de los mercados discográficos internacionales, se verifica que la fijación de precios ha sido artificial y abusiva por parte de las grandes compañías discográficas ${ }^{3}$. Por ejemplo, en los últimos diez años, el precio de un $\mathrm{CD}$ álbum de lanzamiento se situaba entre los $€$ 20 y $€ 23$, mientras que en 2005 , un CD se sitúa en torno a los $€ 15$; mientras que el precio de los discos de las Majors suele ser más elevado que el precio de los discos lanzados por las Indies (Buquet, 2002; SGAE, 2005).

\footnotetext{
${ }^{3}$ Entre los años 2000 y 2001, tanto en EE.UU. como en la UE, los tribunales de la competencia correspondientes han investigado y concluido que las grandes compañías discográficas se ponían de acuerdo para imponer precios mínimos de venta a las tiendas minoristas.
} 
Las consecuencias de esta estructura oligopólica de la industria de la música y del mercado discográfico español son las mismas que las comentadas en relación a la industria de la música a nivel internacional. Esto es, el desarrollo por parte de las Majors de un modelo de explotación global de unos pocos productos musicales de éxito y, a la vez, la domesticación y explotación de los repertorios musicales locales, compitiendo en los mercados nacionales, alimentándose de los pequeños sellos independientes y disminuyendo la participación de las PYMED.

Por último, un mercado discográfico con estas características, en el que la diversidad de productos disponibles se ve disminuida, y desde las estrategias de lanzamientos, pasando por la fijación de precios, su distribución, su difusión y hasta su consumo están planificados desde las grandes compañías discográficas, más la expansión de las nuevas redes, tecnologías y soportes digitales que facilitan el acceso y la distribución libre de todo tipo de productos musicales, todo ello ha generado las condiciones para el surgimiento de una práctica social muy extendida denominada comúnmente "piratería" de productos musicales.

\section{La piratería musical en España}

En los últimos años, en España se ha desatado un amplio debate social en torno a la cuestión de la "piratería" musical, pero este ha sido un debate muy ideologizado desde el comienzo, y actualmente es difícil discernir qué es piratería de lo que no lo es, cuál es su impacto real sobre la industria de la música y hasta qué punto se utiliza este término para defender ciertos intereses de las partes en conflicto.

Según la definición de la IFPI, piratería es la infracción deliberada de la ley de copyright a escala comercial, y en relación a la piratería de productos musicales, esto es la copia no autorizada de una obra original con propósitos comerciales sin el consentimiento del propietario de los derechos sobre la misma ${ }^{4}$ Y según la definición de la SGAE, se entiende por piratería cualquier acto por el cual se lleva a cabo una explotación (esto es, de

\footnotetext{
4 "The term of piracy is generally used to describe the deliberate infringement of copyright on a commercial scale. In relation to the music industry... simple piracy is the unauthorised duplication of an original recording for commercial gain without the consent of the rights owner." (IFPI).
} 
acuerdo al diccionario de la RAE: "sacar utilidad de un negocio o industria en provecho propio") de derechos de propiedad intelectual de manera ilícita, con la finalidad de eludir el cumplimiento de la ley.

En relación a la piratería clásica de los CD musicales, según el último informe de la IFPI, el volumen de los CD pirateados en todo el mundo pasó de 640 millones en el año 2000 a 1.155 millones en 2004, aunque el ritmo de crecimiento se ha ralentizado en los dos últimos años. En 2004, el volumen de negocio de la piratería de CD se estimó en U\$ 4.600 millones, el equivalente al 34\% de las ventas legales de CD (IFPI, 2005a).

En el caso particular de España, en 2004 se vendieron 50 millones de discos y se facturaron 428,47 millones de euros, mientras que el número de CD piratas vendidos fue de 16 millones con una facturación de 64,83 millones de euros o el equivalente al 24\% del mercado legal de CD. España se encuentra así entre los cinco países de la Unión Europea con un nivel de piratería que ronda entre el 10\% y el 24\% de su mercado legal, junto a Bélgica, Finlandia, Holanda y Eslovenia (IFPI, 2005a).

En relación a la distribución y descarga de archivos musicales en Internet, según los datos de la IFPI, en enero de 2006 el número de archivos musicales disponibles en Internet para ser distribuidos y descargados era de 885 millones, de los cuales 775 millones estaban disponibles en las redes de usuarios P2P y el resto de archivos en páginas web. Esta cifra es ligeramente superior a la registrada en enero de 2005 (870 millones) pero inferior a la registrada en junio del mismo año (900 millones), y desde el pico alcanzado en abril de 2003 con 1.1 milliardos de archivos disponibles, la caída registrada hasta hoy ronda el 20\% (IFPI, 2006).

Según el mismo informe, en el Reino Unido y Alemania, el número de usuarios de Internet que descarga música de forma legal ya supera a aquellos que descargan música de forma ilegal, y en Europa el número de usuarios que compra música de forma regular en Internet ronda el 4\%, mientras que aquellos que descargan música de forma ilegal llega al 6\%. La IFPI estima que de cada cinco compradores de música en Internet solo uno es usuario de las redes P2P, y que el 25\% de los usuarios de estas redes estaría dispuesto a pagar por los archivos musicales si los servicios comerciales fueran mejores, mientras que 
un 50\% no estaría dispuesto aún a pagar por ellos; y según otras estimaciones, un 43\% de los usuarios de Internet que descargan música lo hacen a través de las plataformas comerciales (Pew Internet, 2005). La IFPI asegura por otra parte que los usuarios dispuestos a descargar música de forma legal irán superando a aquellos que lo hacen de forma ilegal (IFPI, 2006).

En el caso de España, según el informe de la OCDE, los usuarios de sistemas P2P representan el 1.1\% del total de usuarios de estos sistemas en el mundo (OECD, 2005); y de acuerdo al último informe de la Encuesta General de Medios en España, el 23.2\% de los usuarios españoles de Internet utiliza algún sistema P2P para descargar música (AIMC, 2005).

Ahora bien, sobre este fenómeno económico, político y sociocultural tan reciente y complejo, tanto en su vertiente off line como on line, es necesario hacer algunas distinciones y tomar en cuenta no sólo las interpretaciones de la propia industria discográfica sino también las de otras instituciones e investigadores.

Es evidente que la conformación de un mercado ilegal y paralelo al mercado legal afecta a la venta legal de los $\mathrm{CD}$, genera pérdidas de empleos en el sector de la música y no aporta impuestos al sector público, y por esto la piratería comercial es una actividad que debe ser combatida. Pero también es evidente que la copia no autorizada de productos culturales muchas veces beneficia a sectores sociales con escasos recursos que, de otra forma, no accederían a ellos dados sus precios prohibitivos.

No obstante, el estudio del impacto económico y sociocultural de la "piratería on line" sobre la industria de la música es muy complejo y controvertido. Según el informe de la OECD (2005), la caída de las ventas de fonogramas en los últimos años está relacionada con una multiplicidad de factores, y la descarga de música en Internet por sí sola no es una causa determinante. Además de los factores señalados en relación al declive del soporte CD y su reemplazo por nuevos soportes digitales y la piratería comercial de $\mathrm{CD}$, existen una multiplicidad de factores relacionados tanto con los cambios en la oferta como con las transformaciones en el comportamiento de la demanda de música (Vogel, 2001). 
Incluso la propia industria de la música ha señalado que el impacto de la descarga de música en Internet es muy difícil de cuantificar y ha enumerado otros factores adicionales para explicar la caída de ventas de música, tales como el agotamiento de ciertos repertorios musicales, los errores en las estrategias de marketing, promoción y distribución de las compañías discográficas, y fundamentalmente el aumento de la competencia con otros sectores de las I.C. por los recursos económicos que las personas destinan a los productos de ocio y entretenimiento (OECD, 2005).

Uno de los efectos negativos a tener en cuenta de la piratería, tanto en su vertiente analógica como digital, no es precisamente la supuesta pérdida de ganancias de las compañías discográficas y sus artistas "estrella", sino la pérdida de mercado de las pequeñas compañías discográficas dado que con la piratería se difunden principalmente los productos musicales de éxito a nivel internacional, más las pérdidas de empleos dentro del sector de la música y las pérdidas de impuestos para el sector público (Yúdice, 2002).

Por una parte, si bien es cierto que la piratería pone los CD musicales al alcance de los sectores con menos recursos económicos, por otra, es evidente que esto no resuelve el problema fundamental de la participación de estos sectores en la producción cultural. Y en este sentido, el acceso al consumo cultural no resuelve la cuestión de la diversidad en la producción cultural, y por ello la piratería no constituye "la solución" frente al dominio de las Majors (Canclini y Moneta, 1999; Smiers, 2003; Yúdice, 2002).

Aún así, las reacciones de la propia industria discográfica han promovido aún más las prácticas de piratería musical, criminalizando a miles de usuarios por el intercambio y la descarga de música en Internet y por la realización de copias privadas de CD, pero sin atender el reclamo de estos usuarios, entre otros, en relación a la escasa oferta musical alternativa y a los precios abusivos que imponen las compañías discográficas.

Es indudable que la piratería de productos musicales, ya presente en el entorno off line y multiplicada a una escala inusitada en el entorno on line, vulnera el derecho de los autores y creadores a gestionar la distribución y reproducción de sus obras musicales; pero también es incuestionable que la organización oligopólica de la industria de la música y el sistema de copyright no facilitan la promoción de esos derechos. 
La solución a este grave conflicto de intereses no pasa ni por defender una supuesta "gratuidad" de los productos musicales en Internet, ni por la restricción al acceso de los mismos y menos aún por la "criminalización" y la acción represiva contra miles de ciudadanos. Antes bien, se impone la necesidad de un nuevo pacto social que reconcilie el derecho de los autores a vivir de su trabajo con el derecho de acceso universal a la cultura como un recurso de dominio público (Quéau, 2000), que redunde en beneficio de los creadores musicales, de las pequeñas y medianas empresas discográficas y del público en general, y no sólo en beneficio de las grandes compañías discográficas y de las asociaciones que las representan (Bustamante y otros, 2003).

Por último, el rechazo absoluto a las nuevas formas de distribución, acceso y consumo de productos musicales por su presunto atentado contra la propiedad intelectual es una manifestación más de la ideología del "todo mercado", que no tolera el surgimiento de espacios alternativos de acceso a la producción musical, y por lo tanto de promoción y supervivencia de artistas, creadores y pequeñas y medianas empresas discográficas (Bustamante y otros, 2003).

\section{Conclusiones}

Las principales consecuencias de un mercado discográfico con estas características, como el mercado español, en el que las Majors se alimentan de algunos productos musicales procedentes de los pequeños sellos discográficos pero controlan la distribución y explotación de los mismos a escala masiva, son el deterioro del "efecto catálogo" tradicional de la industria de la música, un mayor condicionamiento de los gustos de los consumidores hacia unos pocos productos musicales de éxito, y fundamentalmente un daño importante en el tejido industrial de las PYMED nacionales.

Por otra parte, en los últimos años la industria discográfica viene experimentando una crisis debido a una multiplicidad de factores, entre los que se incluyen: la competencia del sector musical por los recursos económicos que los ciudadanos destinan al consumo cultural en general, el declive de los soportes tradicionales de la música como el CD, el recambio de estos por nuevos soportes digitales, la copia privada de discos conjuntamente 
a la descarga de música en Internet, más el agotamiento de algunos repertorios musicales y el aumento abusivo de los precios de los discos por parte de las Majors.

En este contexto, la expansión de nuevas redes y tecnologías digitales facilitó el surgimiento de nuevas formas de acceso y consumo musical por parte de una amplia mayoría de ciudadanos, frente a lo cual la industria discográfica reaccionó intentando reprimir estas prácticas sociales, generando así mayores resistencias por parte de los consumidores de música a seguir pagando por algo que podían obtener gratuitamente o a muy bajo costo.

Asimismo, las grandes compañías discográficas están desarrollando nuevos modelos de distribución y comercialización de música en Internet y a través de la telefonía móvil, principalmente, lo cual está relanzando el negocio de la música hacia nuevos mercados digitales emergentes.

No obstante, se verifica que al igual que el mercado de la música off line, el mercado de la música on line presenta una estructura oligopólica donde la distribución de música se concentra en dos grandes plataformas de distribución de música (Apple y Napster) a través de las cuales las Majors comercializan sus productos musicales de mayor éxito, y en las que la presencia de los productos musicales procedentes de las PYMED es prácticamente inexistente.

En este sentido, vemos que la supuesta "revolución digital" en la industria de la música parece ser la continuidad de la misma estructura oligopólica del mercado de la música tradicional a través de los nuevos medios y tecnologías digitales.

Por esto, entendemos que no son las nuevas tecnologías y las redes digitales los posibles agentes de un cambio en la industria de la música, aunque tampoco el comportamiento de los ciudadanos al desarrollar nuevas formas de acceso y consumo musical.

Vemos que las posibilidades de transformación de la industria de la música radican fundamentalmente en las políticas culturales públicas que puedan implementarse desde los distintos Estados nacionales, tendentes a recomponer el tejido de las Industrias Culturales 
nacionales y locales, aumentando la participación de las Pequeñas y Medianas Empresas

Discográficas y promoviendo la pluralidad de la oferta cultural en general.

\section{Bibliografía:}

AIMC. (2005). Encuesta General de Medios (EGM): La audiencia de Internet octubre/noviembre 2005. Madrid: Asociación para la Investigación de Medios de Comunicación (AIMC).

Buquet, G. (2002). La industria discográfica: reflejo tardío y dependencia del mercado internacional. En E. Bustamante (Ed.), Comunicación y cultura en la era digital. Industrias, mercados y diversidad en España. (Vol. I). Madrid: Gedisa.

Bustamante, E., y otros. (2003). Hacia un nuevo sistema mundial de comunicación. Las industrias culturales en la era digital. (Vol. 2). Barcelona: Gedisa.

Canclini, N. G., y Moneta, C. (1999). Las industrias culturales en la integración latinoamericana. Buenos Aires: EUDEBA.

IFPI. (2005a). The Recording Industry 2005. Commercial piracy report. London: IFPI Market Research Publications.

IFPI. (2005b). The Recording Industry in numbers 2005. London: IFPI Market Research Publications.

IFPI. (2006). Digital Music Report 2006. London: IFPI Market Research Publications.

OECD. (2005). Report on the digital music industry. Organisation for Economic Cooperation and Development. Consultado el: 1/01/2006, en la página web: http://www.oecd.org/document/46/o,2340,en $2649 \quad 3744134994926 \quad 1 \quad 1$ $137441,00 . h t m l$

Pew Internet. (2005). Music and Video downloading moves beyond P2P. Pew Internet. Consultado el: 1/3/2006, en la página web: www.pewinternet.org/pdfs/PIP Filesharing_March05.pdf

Promusicae. (2005). Libro Blanco de la música en España. Madrid: Productores de Música de España.

Quéau, P. (2000). La planète des esprits. París: Editions Odile Jacob.

SGAE. (2004). La SGAE reparte entre sus socios 295 millones en 2004, un 7\% más. Sociedad General de Autores y Editores de España. Consultado el: 1/1/2006, en la página web:

http://www.sgae.es/contenido/cont.inm?instanceId=989\&tipoId=38\&selecte $\mathrm{dMenu}=29$

SGAE. (2005). Anuario SGAE de las Artes Escénicas, Musicales y Audiovisuales. Madrid: Sociedad General de Autores y Editores de España. 
Smiers, J. (2003). Regulations in favour of cultural diversity. Cultural Centre De Balie: Conference Regulations in favour of Cultural Diversity. Consultado el: 16/12/2003, en la página web: http://www.hku.nl/usa/centres/centresen/cvo/papers smiers/paper\%20and\%20comments.doc.pdf

Vogel, H. L. (2001). Entertainment Industry Economics. A Guide for Financial Analysis. Cambridge: Cambridge University Press.

Yúdice, G. (2002). Las industrias culturales: más allá de la lógica puramente económica, el aporte social. OEI, Pensar Iberoamérica (Revista de Cultura de la Organización de los Estados Iberoamericanos). Consultado el: 16/12/2003, en la página web: http://www.campus-oei.org/pensariberoamerica 